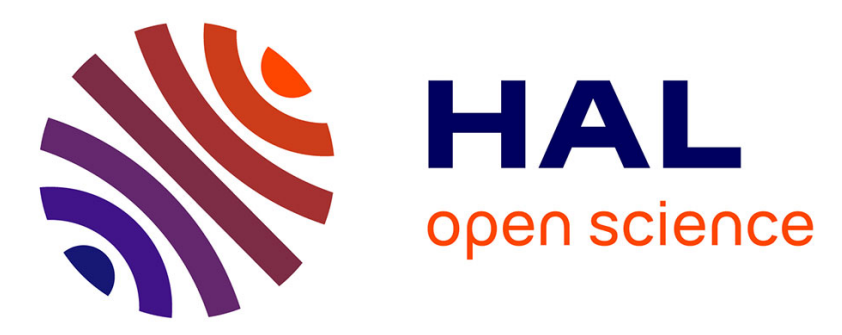

\title{
Three-dimensional transesophageal echocardiography for descending aortic atheroma: a preliminary study
}

Nadjib Hammoudi, Malek Ihaddaden, Sylvie Lang, Florent Laveau, Stephane Ederhy, Pierre-Louis Michel, Sonia Alamowitch, Ariel Cohen

\section{- To cite this version:}

Nadjib Hammoudi, Malek Ihaddaden, Sylvie Lang, Florent Laveau, Stephane Ederhy, et al.. Threedimensional transesophageal echocardiography for descending aortic atheroma: a preliminary study. International Journal of Cardiovascular Imaging, 2014, 30, pp.1529-1537. 10.1007/s10554-014-05020. hal-01102908

\section{HAL Id: hal-01102908 https: / hal.sorbonne-universite.fr/hal-01102908}

Submitted on 13 Jan 2015

HAL is a multi-disciplinary open access archive for the deposit and dissemination of scientific research documents, whether they are published or not. The documents may come from teaching and research institutions in France or abroad, or from public or private research centers.
L'archive ouverte pluridisciplinaire HAL, est destinée au dépôt et à la diffusion de documents scientifiques de niveau recherche, publiés ou non, émanant des établissements d'enseignement et de recherche français ou étrangers, des laboratoires publics ou privés. 


\title{
ORIGINAL INVESTIGATIONS
}

\section{Three-Dimensional Transesophageal Echocardiography for Descending Aortic Atheroma: A Preliminary Study}

\author{
Nadjib Hammoudi, MD, Malek Ihaddaden, MD, Sylvie Lang, PhD, Florent Laveau, MD, \\ Stephane Ederhy, MD, Pierre-Louis Michel, MD, Sonia Alamowitch, MD, PhD, Ariel Cohen, MD, PhD
}

From the Department of Cardiology, Assistance Publique - Hôpitaux de Paris, Pitié-Salpêtrière Hospital, Paris, France (N.H., F.L., P.-L.M.); Department of Cardiology, Assistance Publique Hôpitaux de Paris, Ambroise Pare Hospital, Boulogne-Billancourt, France (M.I.); Department of Cardiology, Assistance Publique - Hôpitaux de Paris, Saint Antoine Hospital, Paris, France (S.L., S.E., A.C.); Department of Neurology, Assistance Publique - Hôpitaux de Paris, Tenon Hospital, Paris, France (S.A.); University Paris 6, Faculté de Médecine Pierre et Marie Curie, Paris, France (N.H., F.L., P.-L.M., S.A., A.C.); Paris lle de France Ouest University, Versailles, France (M.I.).

Short title: 3D Echocardiography for Descending Aortic Atheroma

Address for correspondence: Nadjib Hammoudi, Institut de Cardiologie, Groupe Hospitalier PitiéSalpêtrière, Assistance Publique - Hôpitaux de Paris, 47-83, boulevard de l'Hôpital, 75651 Paris Cedex 13. E-mail: nadjib.hammoudi@psl.aphp.fr.

Telephone: +3314216 5535. Home telephone: +33 607709254 . 


\section{ABSTRACT}

Purpose: Transesophageal echocardiography (TEE) is an efficient method for characterization of aortic atherosclerotic plaques (AAP). The aim of our study was to evaluate the feasibility and the additional contribution of three-dimensional (3D) TEE in the evaluation of AAPs in descending thoracic aorta.

Methods: We studied 82 patients referred for TEE regardless of the indication. All patients underwent two-dimensional (2D) conventional acquisitions. A 3D TEE study was performed for all AAPs localized in the descending thoracic aorta. Thickness, degree of calcification, the presence of ulceration or mobile debris were compared for $2 \mathrm{D}$ and $3 \mathrm{D}$ modes. From 3D data, three types of AAPs were defined according to their morphological characteristics (surface and contours).

Results: Among 192 AAPs found on 2D acquisition, 189 (98.4\%) were also identified by 3D TEE. For AAP characterization, agreement was good between $2 \mathrm{D}$ TEE and 2D extracted from 3D with the multiplanar reconstruction mode: $83.6 \%(k=0.69)$ for thickness and $82.5 \%(k=0.72)$ for degree of calcification. All AAPs ulcerations $(n=13)$ and mobile debris $(n=3)$ seen in 2D were identified in 3D. 2D characteristics of the 3D AAPs' morphological types were different: type I plaques were thin and rarely calcified; type III plaques were thicker and often calcified; and type II presented intermediate characteristics. There was overlap among groups.

Conclusion: 3D TEE is a feasible method for the analysis of AAPs. In addition to conventional characterization, 3D TEE provides a new morphological approach to AAPs.

Keywords: 3D TEE, Aortic plaque, Atheroma, Imaging, Transesophageal echocardiography 


\section{BACKGROUND}

Atherosclerotic disease is a leading cause of cardiovascular death [1] and constitutes a major public health issue. This disease, in its different localizations, is therefore generating increasing interest and remains a diagnostic and therapeutic challenge.

In recent years, significant progress has been made in the examination of atherosclerotic plaque due to improvements in imaging methods. Several techniques for the morphological and functional analysis of atherosclerotic plaques were developed for coronary artery disease,[2][3]and magnetic resonance imaging (MRI) provides new data on carotid plaques.[4]

Atherosclerosis in the descending thoracic aorta is a marker of diffuse atherosclerotic cardiovascular disease; atherosclerotic plaques localized in this segment of aorta are associated with coronary artery disease and cardiovascular events.[5][6][7][8]Transesophageal echocardiography (TEE) is an established technique to study aortic atherosclerotic plaques (AAP) [9][10]; moreover descending aortic atheroma is the most common extracardiac finding during routine TEE regardless of the indication.[11]

Three-dimensional (3D) TEE was proposed and validated as a complementary ultrasound exploration to the two-dimensional (2D) approach, its utility in various circumstances including native and prosthetic valve analysis and for monitoring of percutaneous catheter-based interventions has been established.[12]However, the usefulness of 3D TEE in the examination of AAPs has not been yet well explored.[13]

The objective of this study was to evaluate the feasibility and the contribution of 3D TEE in the assessment of AAPs of the descending thoracic aorta.

\section{METHODS \\ Study Population}

From January 1 st to June 30th 2010, all patients referred to the ultrasound laboratory for TEE and examined by the same experienced operator (N.H.), who was familiar with the 3D mode, were considered for the study. The examination was carried out according to a standardized protocol. Exclusion criteria were the classic contraindications for TEE,[10][14] the absence of aortic atheroma identified in 2D TEE, and an incomplete examination of the aorta due to poor tolerance of the examination (Figure 1). 
Ninety three patients were considered for the study. Of these, 11 did not meet all inclusion and exclusion criteria. The remaining 82 patients constituted the study population. Data on diabetes, hypertension, dyslipidemia, smoking, and serum creatinine level were collected from medical records. The consent of all patients was obtained and the study was approved by the institutional committee on human research.

\section{Acquisition of Images}

All examinations were performed on an iE33 system (Phillips Medical Systems, Andover, MA, USA) equipped with a 3D TEE probe. After a complete transthoracic echocardiogram, local anesthesia of the pharynx by lidocaine, and mild sedation with midazolam (mean dose $1.3 \pm 0.4 \mathrm{mg} ; 5$ patients were not sedated), patients were placed in the left lateral decubitus position. Following a conventional 2D TEE examination, [10][14] in particular of the valves, the left atrium, and the interatrial septum, the operator focused on the aorta. To optimize image quality, the size of the aorta on the display was increased by reducing the depth, the focus was moved to the near field, and the transducer frequency was increased. [10] The probe was withdrawn very gradually until the aortic arch in its various segments was reached.[9]The descending thoracic aorta was visualized in its short axis (TEE at $0^{\circ}$ ), and whenever an AAP (defined as a hyperechoic thickening of the intima, regardless of the thickness) was identified in $2 \mathrm{D}$ images, a rotation of $90^{\circ}$ was carried out for viewing on its long axis and a 3D zoom mode acquisition was performed. Image acquisition was standardized, alternating a 2D loop of an AAP followed systematically by a 3D loop of the same AAP. Thus, 2D and 3D loops of the same AAP followed each other during the recording of the images.

\section{Image Analysis}

The images were transferred to a computer equipped with Phillips QLAB software (version 8.1) for post-processing. The analyses were performed more than 6 months after the examinations by two investigators (N.H., M.I.). The total duration of the TEE (defined as the time between the first and the last image of the examination) and the specific duration of the examination of the aorta (defined as the time between the first and the last image of the thoracic aorta) were collected.

\section{D Data}

2D data were analyzed blinded to 3D TEE images and to clinical data. The number of AAPs was quantified and each plaque was measured on the incidence where it appeared the thickest (mean of 3 
measurements). The AAPs were then classified into three categories according to their thickness: $<1$ $\mathrm{mm}, 1-3.9 \mathrm{~mm}$, and $\geq 4 \mathrm{~mm}$,[9] and the degree of calcification of each AAP was evaluated: not calcified (0), slightly calcified $(+)$, or very calcified $(++)$.The presence of mobile "debris" was noted; a disruption or marked irregularities of the AAPs' surface defined ulceration ( $\geq 2 \mathrm{~mm}$ in depth and width).[9]

Particular attention was given to the precise location of the AAP analyzed in the segment of descending aorta studied. Indeed, it was decided to divide the descending thoracic aorta in TEE $0^{\circ}$ into 4 segments, segments 1 and 2 corresponding to the portions closest to the probe, and segments 3 and 4 corresponding to the other half of the aorta.

\section{D Data}

The 3D analysis was performed blinded to the 2D TEE data to clinical data. The quality of the 3D images was graded as insufficient, good, or optimal. To make sure that the plaque analyzed in 3D was the same which was analyzed in $2 \mathrm{D}$, in cases where several plaques were identified on $3 \mathrm{D}$ acquisition, the elements of localizations defined above and collected during 2D analysis were used to define the plaque to be analyzed in 3D.

\section{Morphological Analysis in 3D}

Initially, 20 3D acquisitions of randomly selected AAPs were analyzed simultaneously by three confirmed echocardiographers (M.I., N.H., A.C. respectively 5, 10 and 25years experience with TEE). By consensus, AAP in 3D was defined as a persistent intimal thickening despite the reduction in gain to zero. In addition, three different morphological types of plaques were identified (Figure 2). Type I atheroma plaque was defined as an intimal thickening with regular contours and a smooth surface. Type II atheroma plaque was defined as an intimal thickening, with irregular contours and a bumpy surface. Type III atheroma plaque was defined as an intimal thickening with strongly marked irregular contours, a tortuous surface and an empty band under the plaque separating it from the aortic wall. In a second step, the morphological study of all 3D acquisitions was carried out by two investigators (N.H., M.I.) and the AAPs identified were classified according to the three types of plaques defined above. 


\section{D reconstructions from 3D Data}

After the 3D morphological analysis, a study in multiplanar reconstruction (MPR) mode, allowing for the extraction of $2 \mathrm{D}$ data from the $3 \mathrm{D}$ volume, was systematically carried out. From reconstructed $2 \mathrm{D}$ images, a measurement of the maximum thickness (mean of 3 measurements) was done, and the degree of calcification of each AAP was evaluated (Figure 3). In addition, to morphological analysis from 3D image, the presence of mobile "debris" and/or ulceration was also searched for carefully in whole AAP 3D volume with MPR mode.

\section{Statistical Analysis}

Continuous variables are presented as medians with interquartile ranges (IQRs). Qualitative variables are presented as counts and percentages. The data were compared using the Fisher and KruskalWallis exact tests. Correlations between the different measures were evaluated using the Pearson correlation test. The agreement between 2D data and 2D data extracted from 3D acquisitions by MPR was evaluated by calculating the Kappa coefficient. Using the Bland-Altman approach, the mean difference and the $95 \%$ limit of agreement was also calculated.

To evaluate intra- and inter-observer agreement in the analysis of plaque morphology in 3D, 40 randomly selected plaques were reviewed blinded to the initial evaluation and the Kappa coefficient was calculated. A $P$ value $<0.05$ was considered statistically significant. STATA software version 11 was used to perform all analyses.

\section{RESULTS}

\section{Population Characteristics}

The characteristics of the population are summarized in Table 1. Patients' median age was 62.3 (55.2-75.1) years and 33 patients (40.2\%) out of a total of 82 were women.

\section{Transesophageal Echocardiography}

The tolerance to the examination was satisfactory in $98.8 \%$ of cases and no complication was observed. The median duration of examination was 9.2 (6.2-12.2) minutes, the median time to examine the aorta was $2.2(1.3-3.7)$ minutes. 


\section{D Analysis}

A total of 192 AAPs were identified by 2D TEE, with a median of 2 (1-3) AAPs per patient. Ninety-five (49.4\%) AAPs were not calcified, 65 (33.9\%) were slightly calcified, and 32 (16.7\%) were very calcified.

The median thickness of the AAPs was $2.0 \mathrm{~mm}(1.1-2.7 \mathrm{~mm}) ; 38(19.8 \%)$ were $<1 \mathrm{~mm}, 132(68.8 \%)$ were $1-3.9 \mathrm{~mm}$, and $22(11.4 \%)$ were $\geq 4 \mathrm{~mm}$. Ulcerations and mobile debris were identified in 13 and 4 AAPs respectively.

\section{D Analysis}

Of the 192 AAPs viewed by 2D TEE, 189 (98.4\%) were identified by 3D TEE. The missed plaques were thin (thickness between 0.5 and $1.2 \mathrm{~mm}$ ) and not calcified in 2D. The mean 3D TEE off-line review time was 2.5 minutes per plaque and the quality of the 3D images was rated as good or optimal in $95 \%$ of the cases.

Of the 189 AAPs identified in 3D, 77 (40.7\%) were a type I, 63 (33.4\%) were a type II, and 49 (25.9\%) were a type III. The 2D TEE characteristics for the three morphological types of AAP in 3D TEE were different (Table 2). Type I AAPs are thin and rarely calcified, type III AAPs are thicker and often calcified, and type II AAPs have intermediate 2D TEE characteristics. The 3D morphology could not be formally predicted based on 2D TEE characteristics, due to significant overlap between the measurements of plaque thickness for types II and III (Figure 4).

There was similar intra- and inter-individual agreement for the 3D morphological analysis of AAPs, with $93 \%$ (kappa=0.88) of AAPs classified in agreement with the first reading.

\section{MPR Analysis of 3D Acquisitions}

A good correlation $(r=0.92, P<0.001)$ was observed between 2D measurements extracted from 3D acquisitions and those carried out on the 2D TEE data. The mean difference between the both methods was $-0.1 \mathrm{~mm}$, the $95 \%$ limits of agreement were -1.4 to $1.2 \mathrm{~mm}$ (Figure 5 ).

Table 3 presents the conventional classification of AAPs according to their 2D TEE characteristics and those extracted from 3D by MPR. The agreement between the two methods was good, at $83.6 \%(\mathrm{kappa}=0.69)$ for the measurements of thickness, and $82.5 \%(\mathrm{kappa}=0.72)$ for the degree of calcification.

All AAP ulcerations $(n=13)$ and mobile debris $(n=3)$ seen in 2D have been identified from 3D images (Figure 6) (Video 1). 


\section{DISCUSSION}

In this study evaluating 3D TEE applied to the study of AAPs in the descending thoracic aorta, we have shown the feasibility of the method and an excellent agreement for AAP characterization between the 2D data extracted from 3D TEE and those measured from conventional 2D. In addition, 3D TEE provides an original morphological approach for the study of AAP.

\section{Feasibility}

We showed that 3D TEE is an appropriate tool for evaluating AAP. Indeed, $98.4 \%$ of plaques viewed in 2D were visualized in 3D. The median 3D TEE examination time seems to be acceptable for routine use, being relatively similar to that for a conventional 2D TEE examination.[15][16]

\section{D TEE Aortic Atherosclerotic Plaques}

With 2D TEE a total of 192 AAPs were identified on the descending thoracic aorta. Echocardiographic studies do not usually report the number of plaques studied by aortic segment.[5][6][7]In 120 consecutive necropsy studies, Khatibzadeh et al.[17]reported a mean of 2.2 plaques per patient, including fatty streaks on the descending thoracic aorta. This result is very similar to our own.

\section{D TEE Aortic Atherosclerotic Plaques}

We found good agreement between the 2D measurements and those extracted from 3D by MPR, which has been validated and routinely used for 3D visualization in computed tomography (CT).[18] However, two cases of clear disagreement between the two methods were observed (figure 5). Because these cases concerned very protrusive aortic plaques $(>6 \mathrm{~mm})$, it is possible that the measurements extracted from 3D were not made at exactly the same level of cut as those carried out on the corresponding 2D images; the MPR mode makes it possible to "navigate" in the entire acquired $3 \mathrm{D}$ volume and to measure the thickest part of the plaque, as well as, to analyze the plaque morphology looking in particular for ulcerations or mobile debris (Figure 6). The optimized crosssectional planes of the heart that provides the $3 \mathrm{D}$ is one of the important advantage of this technology over the 2D, it allows accurate measurements of chamber dimensions as well as improved evaluation of the morphology of different structures. [12]. The analysis of the degree of calcification of the plaques also shows a good agreement between the 2D and 3D analyzed in MPR. 
All AAP ulcerations and mobile debris seen in 2D were identified from 3D images. Similarly, a study reported the ability of 3D TEE for the identification of these morphological AAP characteristics.[19]In that study, the 3D TEE was dedicated to morphological assessment of large plaques without any quantitative evaluation from 3D. In another work, a good agreement between quantitative $2 \mathrm{D}$ and $3 \mathrm{D}$ evaluation of $\mathrm{AAP}$ has been shown. However, the evaluation of AAP calcification as well as the morphological analysis of AAP from 3D were not investigated. [13]

A 3D acquisition offers a much greater volume of data than that acquired with 2D acquisition. In this work, we have systematically acquired $2 \mathrm{D}$ and $3 \mathrm{D}$ images separately. Given the good agreement between the information extracted from the two modes, our findings support that a 3D TEE exploration protocol of descending aortic atheroma appear to be as efficient as the standard protocol. In the new approach, the $2 \mathrm{D}$ is used for guiding $3 \mathrm{D}$ volume acquisitions; however, the technically difficult and time consuming on-line 2D multiplane imaging is avoided.

From a morphological perspective, most of the AAPs were classified as type I. While the three types of plaques in 3D acquisitions had different 2D characteristics, there was significant "overlap" of the $2 \mathrm{D}$ appearance between the plaque categories. It is not therefore possible to predict reliably the $3 \mathrm{D}$ morphology from the $2 \mathrm{D}$ characteristics. The clinical meaning of this new morphological $\mathrm{AAP}$ approach remains to be evaluated.

Type III AAP were associated with an empty band under the plaque giving the impression of a "floating" plaque. In 2D acquisitions, this type of plaque is most often calcified. Thus, the space below the plaque could correspond to a posterior acoustic shadowing.

\section{Potential applications of 3D TEE}

The management of the increased workload of the echocardiography laboratories is a current important issue. [20]As descending aortic atheroma is the most common extracardiac finding during TEE, [11] the implementation of the new 3D protocol in routine appear to be a promising interest of 3D AAP approach. It might be helpful to reduce the examination duration. In addition to the optimization of the laboratory efficiency, this could also improve the patient comfort.

3D TEE might also be used to check carefully off-line by MPR mode the entire plaque volume and make sure that the measure of thickness is done at the right place (maximal thickness) with the 
right method (perpendicularly to the aortic wall). That could be very helpful to verify the appropriate classification of the AAP with a 2D thickness close to the $4 \mathrm{~mm}$ validated cut-off value. [9]

The standardization of on-line acquisition protocol and MPR post-processing that allow 3D TEE make this technology a promising tool for clinical research. MRI and CT could also be used for the assessment of AAP. However, MRI is expensive and cannot be performed at the bedside, while CT exposes patients to radiation and contrast injection.

\section{Limitations}

The review time for 3D acquisitions is an obstacle to the use of this technique. However, improved post-processing software should reduce the analysis time in the future.

In this study, the quantitative approach to AAP volume was not possible. Improved postprocessing software should soon allow for this to be evaluated. Recently, a study reported a quantitative approach for AAP volume, which correlated well with 2D measurements. However, in this work the volume was not measured; rather, it was estimated by multiplying the surface area by plaque thickness, which no doubt explains the correlation with 2D acquisitions.[13] Using a pre-commercial new software another group have reported very recently the feasibility of semiautomated volume quantification of AAP from 3D TEE images. [21] In this latter work the correlation between maximum AAP thickness and volume was only moderate. Further studies are warranted to investigate the clinical impact of this very promising new quantitative approach of AAP.

The study of AAP in aortic arch involved in neurovascular disease was not carried out in this study. In fact, we decided as a first step to evaluate 3D TEE approach of AAP in descending thoracic aorta. Our very encouraging results need to be confirmed in aortic arch which is technically more complex to study by ultrasound.

Finally, in this feasibility study we excluded the subjects without aortic atheroma in 2D. It would be interesting in next studies to assess if 3D TEE could detect AAPs that are not detected in 2D. 


\section{Conclusion}

3D TEE is a feasible method for the analysis of AAP of the descending thoracic aorta. In addition to conventional characterization, 3D TEE offers a promising approach to the disease as much morphologically as quantitatively. The clinical impact of 3D TEE approach of AAP should be investigated in larger studies. 


\section{DISCLOSURES}

The authors have no disclosures

FUNDING, GRANT OR OTHER SUPPORT

None

\section{ACKNOWLEDGMENT}

Sophie Rushton-Smith, $\mathrm{PhD}$, provided editorial assistance including editing, checking content and language, formatting, and referencing, and was funded by the authors. 


\section{REFERENCES}

1. Go AS, Mozaffarian D, Roger VL, et al. (2014) Executive summary: heart disease and stroke statistics--2014 update: a report from the American Heart Association. Circulation 129:399-410. doi: 10.1161/01.cir.0000442015.53336.12

2. Gould KL, Johnson NP, Bateman TM, et al. (2013) Anatomic versus physiologic assessment of coronary artery disease. Role of coronary flow reserve, fractional flow reserve, and positron emission tomography imaging in revascularization decision-making. J Am Coll Cardiol 62:1639-1653. doi: 10.1016/j.jacc.2013.07.076

3. Waksman R, Kitabata H, Prati F, et al. (2013) Intravascular ultrasound versus optical coherence tomography guidance. J Am Coll Cardiol 62:S32-40. doi: 10.1016/j.jacc.2013.08.709

4. Takaya N, Yuan C, Chu B, et al. (2006) Association between carotid plaque characteristics and subsequent ischemic cerebrovascular events: a prospective assessment with MRI--initial results. Stroke 37:818-823. doi: 10.1161/01.STR.0000204638.91099.91

5. Varga A, Gruber N, Forster T, et al. (2004) Atherosclerosis of the descending aorta predicts cardiovascular events: a transesophageal echocardiography study. Cardiovasc Ultrasound 2:21. doi: $10.1186 / 1476-7120-2-21$

6. Gu X, He Y, Li Z, et al. (2011) Relation between the incidence, location, and extent of thoracic aortic atherosclerosis detected by transesophageal echocardiography and the extent of coronary artery disease by angiography. Am J Cardiol 107:175-178. doi: 10.1016/j.amjcard.2010.09.003

7. Khoury Z, Schwartz R, Gottlieb S, et al. (1997) Relation of coronary artery disease to atherosclerotic disease in the aorta, carotid, and femoral arteries evaluated by ultrasound. Am J Cardiol 80:1429-1433.

8. Roos CJ, Witkowska AJ, de Graaf MA, et al. (2013) Association of atherosclerosis in the descending thoracic aorta with coronary artery disease on multi detector row computed tomography coronary angiography in patients with suspected coronary artery disease. Int $\mathrm{J}$ Cardiovasc Imaging 29:1829-1837. doi: 10.1007/s10554-013-0266-y

9. Cohen A, Tzourio C, Bertrand B, et al. (1997) Aortic plaque morphology and vascular events: a follow-up study in patients with ischemic stroke. FAPS Investigators. French Study of Aortic Plaques in Stroke. Circulation 96:3838-3841. 
10. Hahn RT, Abraham T, Adams MS, et al. (2013) Guidelines for Performing a Comprehensive Transesophageal Echocardiographic Examination: Recommendations from the American Society of Echocardiography and the Society of Cardiovascular Anesthesiologists. Journal of the American Society of Echocardiography 26:921-964. doi: 10.1016/j.echo.2013.07.009

11. Alkhouli M, Sandhu P, Wiegers SE, et al. (2014) Extracardiac Findings on Routine Echocardiographic Examinations. J Am Soc Echocardiogr. doi: 10.1016/j.echo.2014.01.026

12. Lang RM, Badano LP, Tsang W, et al. (2012) EAE/ASE Recommendations for Image Acquisition and Display Using Three-Dimensional Echocardiography. Journal of the American Society of Echocardiography 25:3-46. doi: 10.1016/j.echo.2011.11.010

13. Aggeli C, Kazazaki C, Felekos I, et al. (2011) Role of real time-3D transesophageal echocardiography in evaluating the atheromatous burden of thoracic aorta in patients with heterozygous familial hypercholesterolemia: comparison with 2D transesophageal study. Int J Cardiol 150:92-93. doi: 10.1016/j.jijcard.2011.03.035

14. Flachskampf FA, Badano L, Daniel WG, et al. (2010) Recommendations for transoesophageal echocardiography: update 2010. Eur J Echocardiogr 11:557-576. doi: 10.1093/ejechocard/jeq057

15. Daniel WG, Erbel R, Kasper W, et al. (1991) Safety of transesophageal echocardiography. A multicenter survey of 10,419 examinations. Circulation 83:817-821.

16. Min JK, Spencer KT, Furlong KT, et al. (2005) Clinical features of complications from transesophageal echocardiography: a single-center case series of 10,000 consecutive examinations. J Am Soc Echocardiogr 18:925-929. doi: 10.1016/j.echo.2005.01.034

17. Khatibzadeh M, Mitusch R, Stierle U, et al. (1996) Aortic atherosclerotic plaques as a source of systemic embolism. J Am Coll Cardiol 27:664-669.

18. Rothman SL, Geehr RB, Kier EL, Hoffman HB (1978) Multiplanar reconstruction as an aid in CT diagnosis. Neuroradiology 16:596-597.

19. Haruki N, Takeuchi M, Kaku K, et al. (2010) Prevalence and clinical implication of complex atherosclerotic plaque in the descending thoracic aorta of Japanese patients assessed by transesophageal echocardiography. Circ J 74:2627-2632.

20. Hammoudi N, Duprey M, Régnier P, et al. (2014) Pretest probability of a normal echocardiography: validation of a simple and practical algorithm for routine use. Arch Cardiovasc Dis 107:105-111. doi: 10.1016/j.acvd.2014.01.004 
21. Piazzese C, Tsang W, Sotaquira M, et al. (2014) Semiautomated detection and quantification of aortic plaques from three-dimensional transesophageal echocardiography. J Am Soc Echocardiogr 27:758-766. doi: 10.1016/j.echo.2014.03.003 


\section{FIGURE LEGENDS}

Figure 1. Study flow chart.

AAP, aortic atherosclerotic plaque; MPR, multiplanar reconstruction mode; TEE, Transesophageal echocardiography; TTE, transthoracic echocardiography

Figure 2. Three morphological types of plaques found by 3D TEE (arrows):

Type I: Intimal thickening, regular contours, and smooth surface.

Type II: Intimal thickening, irregular contours, and bumpy surface.

Type III: Strongly marked irregular contours with tortuous surface and empty band under the plaque.

Figure 3. MPR allowing the analysis of degree of calcification and the thickness measurement of an aortic atherosclerotic plaque (arrows) from a 3D image.

(A) Long- and (B) short-axis reconstructions; note the hyperechogenic image with a posterior cone shadow (calcified plaque); (C) short-axis reconstruction with thickness measurement; and (D) original 3D morphology.

Figure 4. 2D Thickness values in the three morphological 3D types of plaques. There was an overlap among the groups and the 3D morphology could not be predicted from 2D data. The box defines the interquartile range, the median value is indicated by the bar, and the whiskers indicate the minimal and maximal thickness.

Figure 5. Bland-Altman analysis demonstrated a good agreement between plaque thickness derived from 2D TEE and 3D MPR.

MPR, multiplanar reconstruction mode; SD, standard deviation; TEE, Transesophageal echocardiography

Figure 6. Two short axis reconstruction plans of the aorta extracted with MPR mode from a 3D acquisition of a voluminous aortic plaque. (A) The red line cutting plane shows the maximum thickness of the plaque and an ulceration (arrow); (B) at the green line cutting plane the plaque appear to be thinner and the ulceration is not displayed. 


\section{TABLES}

Table 1 Baseline characteristics of the study population $(n=82)$

\begin{tabular}{lc}
\hline Characteristic & $n(\%)$ or median (interquartile range) \\
\hline Age, years & $62.3(55.2-75.1)$ \\
Gender, women & $33(40.2)$ \\
Body mass index, $\mathrm{kg} / \mathrm{m}^{2}$ & $25.4(22.4-28.0)$ \\
Hypertension & $38 / 77(49.4)$ \\
Smoking (current or past) & $34 / 77(44.2)$ \\
Diabetes & $20 / 77(26.0)$ \\
Hypercholesterolemia & $32 / 77(41.6)$ \\
Atrial fibrillation & $25(30.5)$ \\
Serum creatinine*, $\mu$ mol/L & $77(66-98)$ \\
Prior myocardial infarction & $10 / 77(13.0)$ \\
Prior stroke & $9 / 77(11.7)$ \\
Left ventricular ejection fraction ${ }^{* *}, \%$ & $65(60-70)$ \\
Left atrial area**, cm ${ }^{2}$ & $21(19-28)$ \\
Transesophageal echocardiography indication & $8(8.5)$ \\
$\quad$ Stroke & $6(7.3)$ \\
$\quad$ Atrial fibrillation & $83(9.8)$ \\
$\quad$ Ealvulopathy & \\
Others & \\
\hline & \\
\hline
\end{tabular}

${ }^{*} n=74$; **from transthoracic echocardiography 
Table 2 Two-dimensional (2D) characteristics of the three-dimensional (3D) morphological types of atherosclerotic plaques in the descending aorta $(n=189)$

\begin{tabular}{|c|c|c|c|c|c|}
\hline \multicolumn{2}{|l|}{ Variable } & \multicolumn{3}{|c|}{ From 3D } & \multirow[t]{2}{*}{$P$ value } \\
\hline & & $\begin{array}{l}\text { Type I } \\
(n=77)\end{array}$ & $\begin{array}{l}\text { Type II } \\
(n=63)\end{array}$ & $\begin{array}{l}\text { Type III } \\
(n=49)\end{array}$ & \\
\hline & Plaque thickness, $\mathrm{mm}$ & $1.0(0.8-1.1)$ & $2.3(2.0-3.0)$ & $2.7(2.1-3.6)$ & .0001 \\
\hline \multirow[t]{4}{*}{ From 2D } & Plaque calcification & & & & \\
\hline & Not calcified (0) & $72(93.5)$ & $17(27.0)$ & $3(6.1)$ & \\
\hline & Slightly calcified (+) & $5(6.5)$ & $39(61.9)$ & $21(42.9)$ & $<.0001$ \\
\hline & Very calcified $(++)$ & $0(0)$ & $7(11.1)$ & $25(51.0)$ & \\
\hline
\end{tabular}

Results are expressed as median (IQR) or $\mathrm{n}(\%)$ as appropriate 
Table 3. Agreement analysis between $2 \mathrm{D}$ and $2 \mathrm{D}$ data extracted from 3D images by multiplanar reconstruction mode (MPR)

\begin{tabular}{llcccc}
\hline & & & From 3D & \\
& Thickness & $<1 \mathrm{~mm}$ & $1-3.9 \mathrm{~mm}$ & $\geq 4 \mathrm{~mm}$ & $n$ \\
\hline \multirow{2}{*}{ From 2D } & $<1 \mathrm{~mm}$ & $36(97.3)$ & $1(2.7)$ & $0(0)$ & 37 \\
& $1-3.9 \mathrm{~mm}$ & $23(17.6)$ & $104(79.4)$ & $4(3.0)$ & 131 \\
& $\geq 4 \mathrm{~mm}$ & $0(0)$ & $3(14.3)$ & $18(85.7)$ & 21 \\
\hline & & & From 3D & \\
\hline & Calcification & Not calcified & Slightly calcified & Very calcified & \\
\hline \multirow{2}{*}{ From 2D } & Not calcified & $81(88.0)$ & $11(12.0)$ & $0(0)$ & 92 \\
& Slightly calcified & $3(4.6)$ & $47(72.3)$ & $15(23.0)$ & 65 \\
& Very calcified & $0(0)$ & $4(12.5)$ & $28(87.5)$ & 32 \\
\hline
\end{tabular}

Results are expressed as $\mathrm{n}(\%)$ 